This item was submitted to Loughborough's Research Repository by the author.

Items in Figshare are protected by copyright, with all rights reserved, unless otherwise indicated.

\title{
Lidocaine carboxymethylcellulose with gelatine co-polymer hydrogel delivery by combined microneedle and ultrasound
}

PLEASE CITE THE PUBLISHED VERSION

http://dx.doi.org/10.3109/10717544.2014.935985

\section{PUBLISHER}

(C) Informa Healthcare

\section{VERSION}

AM (Accepted Manuscript)

\section{PUBLISHER STATEMENT}

This work is made available according to the conditions of the Creative Commons Attribution-NonCommercialNoDerivatives 4.0 International (CC BY-NC-ND 4.0) licence. Full details of this licence are available at: https://creativecommons.org/licenses/by-nc-nd/4.0/

\section{LICENCE}

CC BY-NC-ND 4.0

\section{REPOSITORY RECORD}

Nayak, Atul, Hiten Babla, Tao Han, and Diganta Das. 2014. "Lidocaine Carboxymethylcellulose with Gelatine Co-polymer Hydrogel Delivery by Combined Microneedle and Ultrasound”. figshare. https://hdl.handle.net/2134/15548. 
This item was submitted to Loughborough's Institutional Repository (https://dspace.lboro.ac.uk/) by the author and is made available under the following Creative Commons Licence conditions.

\section{creative
commons}

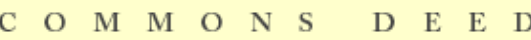

Attribution-NonCommercial-NoDerivs 2.5

You are free:

- to copy, distribute, display, and perform the work

Under the following conditions:

Attribution. You must attribute the work in the manner specified b the author or licensor.

Noncommercial. You may not use this work for commercial purposes.

No Derivative Works. You may not alter, transform, or build upon this work.

- For any reuse or distribution, you must make clear to others the license terms of this work.

- Any of these conditions can be waived if you get permission from the copyright holder.

Your fair use and other rights are in no way affected by the above.

This is a human-readable summary of the Leqal Code (the full license).

\section{Disclaimer 만}

For the full text of this licence, please go to: http://creativecommons.org/licenses/by-nc-nd/2.5/ 
1 Lidocaine carboxymethylcellulose with gelatine co-polymer hydrogel delivery by combined microneedle and ultrasound

\author{
Atul Nayak, Hiten Babla, Tao Han, Diganta Bhusan Das*
} Department of Chemical Engineering, Loughborough University, Loughborough, LE11 3TU, UK ( ${ }^{\star}$ Corresponding Author; Email: D.B.Das@lboro.ac.uk; Tel: 00441509 22509;

Fax: 00441509 223923)

\title{
ABSTRACT
}

A study that combines microneedles and sonophoresis pre-treatment was explored to determine their combined effects on percutaneous delivery of lidocaine from a polymeric hydrogel formulation. Varying ratios of carboxymethylcellulose and gelatine (NaCMC:gel range are 1:1.60-1:2.66) loaded with lidocaine were prepared and characterised for zeta potential and particle size. Additionally, variations in the formulation drying techniques were explored during the formulation stage. Ex-vivo permeation studies using Franz diffusion cells measured lidocaine permeation through porcine skin after pre-treatment with stainless steel microneedles and $20 \mathrm{kHz}$ sonophoresis for 5 and 10 minute durations. A stable formulation was related to a lower gelatine mass ratio because of smaller mean particle sizes and high zeta potential. Lidocaine permeability in skin revealed some increases in permeability from combined microneedle and ultrasound pre-treatment studies. Furthermore, up to 4.8 fold increase in the combined application was observed compared with separate pre-treatments after 30 minutes. Sonophoresis pre-treatment alone showed insignificant enhancement in lidocaine permeation during the initial 2 hours period. Microneedle application increased permeability at a time of $0.5 \mathrm{~h}$ for up to $\sim 17$ fold with an average up to 4 fold. The time required to reach therapeutic levels of lidocaine was decreased to less than 7 minutes. Overall, the attempted approach promises to be a viable alternative to conventional lidocaine delivery methods involving painful injections by hypodermic needles. The mass transfer effects were fairly enhanced and the lowest amount of lidocaine in skin was $99.7 \%$ of the delivered amount at a time of 3 hours for lidocaine NaCMC/GEL 1:2.66 after low frequency sonophoresis (LFS) and microneedle treatment.

Keywords Carboxymethylcellulose, gelatine, microneedles, sonophoresis, lidocaine, percutaneous

Abbreviations: Degree of substitution (D.S.), sodium carboxymethylcellulose ( $\mathrm{NaCMC}$ ), gelatine (gel)

\section{Introduction}

This paper is concerned with the delivery of lidocaine, a common anaesthetic, from a lidocaine carboxymethylcellulose with gel co-polymer hydrogel formulation such as discussed recently by Nayak et al. (2013). An ideal anaesthetic can be described as one that provides rapid, prolonged and effective localised anaesthesia via a mechanism of blocking sensory nerve fibres in the 
periphery that induces no pain and causes no adverse local tissue reaction (Rudin, 2013; Richards and McMahon, 2013; Milewski and Stinchcomb, 2011). Lidocaine hydrochloride is a water soluble weak acid, fully ionised at pH 5.0 and administered into the plasma rich layer under the skin surface (González-Rodríguez et al., 2013; Igaki et al., 2013). However, this administration is conventionally performed via hypodermic needles as a low cost and fast acting method (Kim et al., 2012; Hedge et al., 2011). This is known to cause significant pains (Scarfone et al., 1998). Alternatives, such as eutectic mixture of local anaesthetics (EMLA), a topical form to administer lidocaine, require at least an hour of application to achieve effective analgesia, thus limiting its use especially in emergency situations (Nayak and Das, 2013). Therefore, there are important rationales for the pursuits of alternative lidocaine administration (Nayak et al., 2013; Nayak and Das, 2013). This can be evidenced in the European paediatric drug legislation which backs innovative approaches to develop 'easy to administer' and 'minimally invasive' drug delivery methods (Shah et al., 2011). The alternative rationales for lidocaine delivery include the need for increased safety amongst the patients and healthcare providers, increased compliance with those who possess a fear of needles, reduced discomfort and pain especially in the case of applying anaesthetics as well as improved ease of delivery (Gill and Prausnitz, 2007; Giudice and Campbell, 2006; Li et al., 2010). Oral administration can overcome many of the disadvantages associated with direct injection of drugs (Bal et al., 2010). However, one constraint is low bioavailability of some drugs which limits the effectiveness as therapeutic targets (Shipton, 2012; Benet et al., 1996; De Boer et al., 1979; Huet and Lelorier, 1980). Lidocaine's oral bioavailability is approximately reduced by $65-96 \%$, mainly by hepatic enzymes (Fasinu et al., 2011; Fen-Lin et al., 1993). In principle, innovative percutaneous delivery method could be used to overcome the barriers associated with direct injection and oral administration of drugs (Polat et al., 2011) such as lidocaine. The rate of passive diffusion (PD) of drugs by percutaneous delivery depends on the molecular structure, size and hydrophobicity in conjunction with the drug concentration gradients. However, many studies have used combinations of PD and non-invasive techniques with varying success, e.g., microneedles and ultrasound (Han and Das, 2013; Chen et al., 2010). This is the topic of this paper and it is discussed in more detail below.

65

Microneedles are needle-like structures of the size order of microns commonly arranged in a matrix (Gill and Prausnitz, 2007; Zhang et al., 2014; Olatunji et al., 2014). The geometry of microneedle influences its ability to pierce the skin but importantly, it can be designed to control/optimise the rate of drug delivery. The lidocaine NaCMC/GEL hydrogels pseudoplasticity permits the viscous formulation in allowing seepage into microneedle cavities to bypass the stratum corneum skin layer compared with passive diffusion (Nayak et al., 2013). Research has shown that a significant increase in skin permeability can be achieved when optimised microneedle arrays are used where the important factors include microneedle length, number of microneedles, the length and width aspect ratio and surface area of the microneedle patch (Al-Qallaf and Das, 2008; Al-Qallaf and Das, 
microneedles can be adapted to aid lidocaine delivery yielding many fold increase in delivery rate (Kwon, 2004; Li et al., 2008; Wilson et al., 2008; Kochhar et al., 2013; Ito et al., 2013; Zhang et al., 2012; Zhang et al., 2012; Nayak et al., 2013).

In a recent review paper, Nayak and Das (2013) have discussed the possibility of delivering lidocaine using biodegradable micro-needles. In exploring alternative applications of microneedles, a number of other studies have successfully delivered numerous active molecules using microneedles, e.g., hepatitis B vaccine (Guo et al., 2013), Solaraze ${ }^{\circledR}$ gel in extending pore opening (Ghosh et al., 2013b) and naltrexone co-drug with diclofenac drug (Banks et al., 2013). In another recent study, it has been shown that microneedles can be combined with ultrasound for increasing the delivery rate of a large macromolecular drug (Han and Das, 2013). These studies have further raised the hypothesis that microneedles and ultrasound combination could be used for greater epidermal lidocaine delivery in order to determine the significance of optimum sonophoretic power related the effects on lidocaine permeation.

In this context, it is important to state the classification of sonophoresis which is generally based on the frequency of ultrasound. The low frequency sonophoresis (LFS) is defined to be within the range of $20-100 \mathrm{kHz}$ and the high frequency sonophoresis (HFS) is usually for above $0.7 \mathrm{MHz}$ (Polat et al., 2011). The mechanism by which enhanced permeability is achieved via ultrasound can be linked to a number of physical phenomena including thermal effects, formation of cavitation, mechanical effects and convective localised fluid velocities in skin (Lavon and Kost, 2004). However, in the ultrasound pretreatment experiment, it is generally accepted that inertial cavitation is the largest contributor to the enhancement in skin permeability. It is more so with LFS as shown by Merino et al (2003) due to larger bubble size at low frequency range. Inertial cavitation occurs due to pressure variations induced by ultrasound, resulting in rapid growth and collapse of bubbles formed in the coupling medium. The collapsing of the aforementioned bubbles near skin surface will cause microjets due to asymmetrically release of energy. These micro-jets have been confirmed as the main contributors to the permeability increment (Wolloch and Kost, 2010). The effects of ultrasound have been studied for the enhancement of transdermal lidocaine administration with significant enhancement demonstrated with both pulsed and continuous output mode of LFS (Ebrahimi et al., 2012). However, as far as we are aware of, these techniques are yet to be combined and studied for permeability enhancement levels, particularly for lidocaine.

The potential for adapting microneedles for lidocaine delivery via hydrogel microparticles has been discussed previously with the conclusion that there is significant commercial potential for lidocaine microneedle products (Zhang et al., 2012; Nayak et al., 2013). Polymeric hydrogel microparticles are good for the purpose of controlling spreading (i.e., controllable spreading radius, droplet height and contact angle) of the drug formulation over skin (Nayak et al., 2013). A hydrogel drug vehicle comprises the electrostatic polyionic interaction of a branched structural polymer with a relatively 
115 linear polymer in the formation of a cross-linked matrix to encapsulate Lidocaine molecules (Zhao et

116 al., 2006; Lastumäki et al., 2003).

118 In this particular study, the drug vehicle for lidocaine encapsulation is polyanionic, carbohydrate 119 based NaCMC crosslinked with polycationic, protein based gel in forming a hydrogel (Nayak et al., 120 2013). Previously lidocaine formulation bypassing the stratum corneum (SC) epidermal layer was 121 outlined, the viscoelastic properties in adapting a NaCMC/gel network hydrogel prevents slippage of 122 the drug formulation when applied to the skin and the possibility of non-convective flow through the 123 opened cavities of the skin from microneedle treatment (Milewski and Stinchcomb, 2011; Ghosh et 124 al., 2013a). To try and exploit this potential the main aim of the study is to combine the techniques in 125 microneedle array and ultrasound technology as a pre-treatment to meet the definition of an ideal 126 anaesthetic delivery method. Furthermore, this study will focus on using solid microneedles utilising 127 the 'poke and patch' technique. The main advantage of this approach is the technical simplicity 128 required for reproduction of the required micro-needles leading to reduction of cost. The other major 129 advantage is that an extended release is possible using this approach. A carbohydrate based 130 visceral hydrogel formulation was prepared as a model anaesthetic as this provides flexible 131 properties and ability to encapsulate considerable amounts of liquid drug, lidocaine in this instance 132 (Milewski and Stinchcomb, 2011), as discussed in the following section. Furthermore, the spreading 133 behaviour of the prepared formulation was studied and compared with the spreading behaviour 134 lidocaine solution as a Newtonian liquid. Unlike numerous studies performed using synthetic 135 substrates, this study implements porcine skin as a lipophilic substrate as was attempted by Chow 136 et al. (2008).

\section{2. Materials and methods}

$139 \mathrm{NaCMC}$ and gel emulsion was crosslinked to form hydrogels with encapsulated lidocaine in batch 140 scale production. This formulation setup is highly beneficial because of fairly efficient preparation 141 times in achieving a finished drug formulation and low heat treatment in adaptation of green 142 chemistry.

\subsection{Materials and equipments}

145 Sodium carboxymethylcellulose (D.S. 0.9; M.W. 250kD), sorbitan mono-oleate (SPAN 80), 146 glutaraldehyde $50 \% \mathrm{w} / \mathrm{w}$, paraffin liquid (density range: $0.827-0.89 \mathrm{~g} / \mathrm{ml}$ ), lidocaine hydrochloride 147 (M.W. $288.81 \mathrm{~g} / \mathrm{mol}$ ), methylene blue (50\% v/v) and porcine gelatine (type A) were purchased from 148 Sigma Aldrich Ltd (Dorset, UK). Analytical grade acetic acid, high performance liquid 149 chromatography (HPLC) grade acetonitrile and n-hexane (95\% w/w) were purchased from Fisher 150 Scientific UK (Loughborough, UK). A Gemini-NX column (C18) of particle size $3 \mu \mathrm{m}$ was purchased 151 from Phenomenex (Cheshire, UK) for HPLC instrumentation. Amputated porcine ears (age of pig: 51526 months) were purchased from a local butcher and dissected into $20 \mathrm{~mm} \times 20 \mathrm{~mm}$ squares before 
153 storage at $-20 \pm 1^{\circ} \mathrm{C}$. Also $10 \mathrm{~mm} \times 10 \mathrm{~mm}$ squares of same porcine skin were dissected as a

154 substrate for droplet spreading. Microneedle patch (stainless steel, flat arrow head geometry and $1551100 \mu \mathrm{m}$ length) was purchased from nanoBioSciences (Sunnyvale, CA, USA). Branson Digital

156 Sonifier 450 (Danbury, USA) was chosen as the ultrasound output system. This ultrasound system 157 includes an auto-calibrated transducer and a digital output controller. The frequency of the 158 ultrasound is fixed at $20 \mathrm{kHz}$ but the output powers are adjustable between 4 and $400 \mathrm{~W}$. The 159 equipment for droplet spreading studies were AVT Pike F-032 high performance camera (Allied 160 Vision Technologies UK), Camera i-speed LT high speed video (Olympus, UK),

\subsection{Formulation of lidocaine NaCMC:gel hydrogel}

162 Paraffin oil $(100 \mathrm{ml})$ was sheared continuously for up to $400 \mathrm{rpm}$ in a stirred vessel bought from IKA 163 (Staufen, Germany). Span $80(0.5 \%$ w/w) was dispensed in ambient conditions. To this NaCMC $164(1.24 \% \mathrm{w} / \mathrm{w}$ ) in ultrapure water was added dropwise, and depending on the polymeric ratio (c \% w/w), gel in ultrapure water was also added dropwise at $35-40^{\circ} \mathrm{C}$ (Table 1$)$. A subsequent $\mathrm{pH}$ reduction of the solution to $\mathrm{pH} 4.0$ was performed by the addition of acetic acid $(\sim 3 \% \mathrm{w} / \mathrm{w})$. While shearing at $400 \mathrm{rpm}$, lidocaine $\mathrm{HCl}(2.44 \% \mathrm{w} / \mathrm{w})$ was added dropwise in ultrapure water at $20^{\circ} \mathrm{C}$ into the polymer mixture. The polymeric mixture was then cooled to $5-10^{\circ} \mathrm{C}$ for 30 minutes to initially harden the microparticles. Glutaraldehyde $(0.11 \% \mathrm{w} / \mathrm{w})$ was added to the emulsion as a cross linker. Upon returning to $20^{\circ} \mathrm{C}$ temperature the hydrogel mixture was sheared for 2 hours at approximately $1000 \mathrm{rpm}$ to ensure thorough mixing. The lidocaine $\mathrm{NaCMC} / \mathrm{gel}$ formulation was then left to stand

172 until a distinct w/o boundary was observed after which this formulation was left overnight at $1-5^{\circ} \mathrm{C}$.

173 Excess paraffin liquid was removed via $n$-hexane separation shaking (50 \% v/v); top organic layer was discarded before placing the hydrogel formulation in a vacuum oven (Technico, Fistreem International Ltd, Loughborough, UK) under full vacuum and a temperature of $20^{\circ} \mathrm{C}$ for 8 hours. Following this, the formulation was washed with deionised (DI) water and filtered using commercial filter papers with pore size $6 \mu \mathrm{m}$ (Whatman, Ltd, Oxon, UK) for removal of unbound lidocaine before further characterisation. In the case of F5 residual paraffin and n-hexane were removed by rotary evaporation (Heidolph Instruments, Essex, UK). Similarly, the formulation was washed with DI water

\subsection{Zeta potential of lidocaine NaCMC:gel hydrogel}

183 The zeta potential was measured using a Zetasizer (3000 HSa, Malvern Instruments, 184 Worcestershire, UK). Lidocaine NaCMC/gel $(2.0 \pm 0.5 \mathrm{~g} / \mathrm{ml})$ in DI water was injected into the sample port, temperature maintained at $25.0^{\circ} \mathrm{C}$ and the results were obtained in triplicate. The zeta-potential $(\zeta)$ was measured in terms of electrophoretic mobility $(\mu)$ via an optical technique, and $\zeta(\mathrm{mV})$ (Park et al., 2005) of the diluted hydrogel was computed from the Smoluchowski equation (2) where $\mu$ is referenced with latex $\left(\mathrm{m}^{2} \mathrm{v}^{-1} \mathrm{~s}^{-1}\right), \eta$ is the DI volume viscosity $\left(\mathrm{m}^{2} \mathrm{~s}^{-1}\right), \varepsilon_{0}$ and $\varepsilon_{\mathrm{r}}$ are the permittivity in a vacuum and relative permittivity of DI water as medium respectively (Sze et al, 2003). 


$$
\zeta=\frac{4 \pi \mu \eta}{\left(\varepsilon_{r} \varepsilon_{o}\right)}
$$

\subsection{Viscometric analysis of lidocaine NaCMC:gel hydrogel}

193 A well-mixed sample volume $(25 \mathrm{ml})$ of lidocaine NaCMC/gel hydrogel sample was determined for 194 variations to viscoelastic properties at standard temperatures of $20^{\circ} \mathrm{C}$. An inducing shear rotating 195 viscometer (Viscotester VT550, Haake, Germany) with rotor and cup (NV1) assemblies and a constant ravine of $0.35 \mathrm{~mm}$, in between the assembly was adapted in viscometric analysis. More details on this aspect of our work are presented by Nayak et al. (2013).

\subsection{Optical micrographs of lidocaine NaCMC:gel hydrogel}

Micrographs were obtained using an optical microscope (BX 43, Olympus, Southend-on-sea, UK) and a camera attachment captured coloured still images (Retiga-2000R, QImaging, British Columbia, Canada). Micrographs were pictured in triplicate for each formulation. An image processing software (ImageJ) was adapted in pixel measurement via graticule calibration to interpret particle size diameters from a random selection of 50 microparticles per image. ImageJ is a Java-based open source image processing and analysis program developed at the National Institute of Health (NIH), USA.

\subsection{Controlled release of lidocaine from NaCMC/gel hydrogel}

Lidocaine NaCMC/gel hydrogel $(0.1 \pm 0.05 \mathrm{~g})$ was placed in an amber vial and $25.0 \mathrm{ml}$ of $\mathrm{DI}$ water was dispensed before the sample was placed in a pre-heated thermo-stat bath at $37.0 \pm 0.5^{\circ} \mathrm{C}$ (Grant Instruments, Cambridge, UK). Subsequently $1 \pm 0.0005 \mathrm{ml}$ of heated sample removed by autopipette (Eppendorf, Stevenage, UK), filtered using Nylon membranes (Posidyne, $0.1 \mu \mathrm{m}$ ) and analysed for lidocaine content using HPLC instrumentation. The results were measured in triplicate and the standard deviation from sample mean was taken.

\subsection{Ex vivo skin permeation study of lidocaine NaCMC/gel hydrogel}

217 Jacketed Franz diffusion cells (FDC) (Logan Instruments, New Jersey, USA) were used as 218 previously annotated for determining the ex vivo drug permeation rate through porcine skin (Nayak 219 et al., 2013). Porcine ear skin was used in this analysis because of the histological similarity with 220 human skin. Dissected square skin sections $(20 \times 20 \mathrm{~mm})$ were defrosted at $25^{\circ} \mathrm{C}$ for a maximum

221 time of 1 hour before the commencement of this study. The FDC receptor chamber (5.0 ml) was 222 filled with deionised water and constantly stirred using a magnetic flea. The FDC receptor volume

223 was constantly maintained at $37 \pm 1^{\circ} \mathrm{C}$ through a water jacket. A square section of full thickness skin 224 (subcutaneous fat and connective tissue removed) was placed on the top of the aperture surface of 225 diffusion cell with a diffusion area of $1.33 \mathrm{~cm}^{2}$. The average skin thickness was recorded in the range 226 of $760-787 \mu \mathrm{m}( \pm 25 \mu \mathrm{m})$. The continuous viscoelastic properties of skin are unlikely to allow for 
227 microneedles to penetrate beyond $200 \mu \mathrm{m}$ when considering $1500 \mu \mathrm{m}$ needle length rollers

228 penetrating a depth of $150 \mu \mathrm{m}$ (Roxhed et al., 2007; Badran et al., 2009). The lidocaine NaCMC:gel 229 hydrogel $(0.10 \pm 0.03 \mathrm{~g})$ was placed on to the skin's donor compartment, the split second timer 230 initiated and then the skin was securely clamped with a donor lid. A fixed $1.5 \mathrm{ml}$ receptor volume was 231 syringe removed periodically from the receptor chamber and replaced with $1.5 \mathrm{ml}$ of deionised water.

232 Following this the samples were analysed for free lidocaine using HPLC instrument (Agilent 1100 233 series, Hewlett Packard, U.S.). Similar FDC method was used for all drug release experiments 234 concerning passive diffusion, microneedles only pre-treatment, LFS only pre-treatment, 235 microneedles and LFS pre-treatment. Microneedles were carefully applied to the skin ensuring 236 penetration and held in place using a constant pressure device comprising of a pneumatic piston 237 (0.05MPa) for 3 or 5 minutes. LFS was supplied using a probe set to $20 \mathrm{kHz}$ frequency for $5-10$ 238 minutes. Continuous application of ultrasound was implemented due to no significant difference 239 being observed during pre-treatment applications (Herwadkar et al., 2012). The inter-coupling 240 distance between the skin and probe was set to $2 \mathrm{~mm}$ with coupling medium of deionised water. A 241 minimum lidocaine concentration of $1.5 \mu \mathrm{g} / \mathrm{ml}$ was deduced from literature as the permissible 242 effective drug therapeutic value in plasma (Schulz et al., 2012; Grossman et al., 1969).

\subsection{Spreading of lidocaine NaCMC;gel 1:2.33 across porcine skin}

245 The setup for measurement of spreading radius, droplet height and apparent contact angle of 246 droplet was similar to Chao et al (2014). A square section of porcine skin (10 $\mathrm{mm} \times 10 \mathrm{~mm})$ was 247 placed flat in a closed sample box. A sample droplet $(3.0 \pm 0.5 \mu \mathrm{l})$ was dispensed on the porcine 248 skin, camera frame rate capture of 1.85 frames per second (fps) was maintained and the results recorded. Results were obtained in duplicate for the optimum particle size controlled formulation and compared with a duplicate set of lidocaine solution of the same lidocaine loading weight (2.44 \% wt).

\subsection{Histological study}

253 The determination of microneedle insertion depth into skin by post microneedle treatment of skin was adapted from Cheung et al (2014). First, the skin sample is pretreated using $1100 \mu \mathrm{m}$ microneedle patch for $5 \mathrm{~min}$. Then, the porcine skin sample is stained using methylene blue (50\% $\mathrm{v} / \mathrm{v}$ ) and merged into embedding compound (Bright Cryo-m-Bed, Huntingdon, UK) which is filled in a cuboid mould. The whole sample is then put inside the microtome (Bright Cryostat 5030, Huntingdon, UK) to solidify. The frozen sample is cut into $15 \mu \mathrm{m}$ slices and analysed under the microscope for the histology.

\section{Results and discussions}

262 3.1. Lidocaine NaCMC/gel hydrogel microparticle size diameters and morphology

263 Lidocaine encapsulated hydrogel microspheres based on NaCMC and gelatine were prepared using 264 glutaraldehyde in transforming emulsion droplets to defined microparticles. As the mechanisms for ionic interactions in forming spherical microparticles are known (Gupta et al., 2000; Berger et al., 
266 2004), it is not discussed in detail in this paper. The morphological observations of lidocaine

267 NaCMC:GEL microparticles are spherical, well-formed and slightly agglomerated for a significant 268 number of them (Fig. 1a, 1b). Mean particle size diameters (Table 1) in the formulation ranged from $2695.89-14.60 \mu \mathrm{m}$ depending on the formulation with an increase in mean particle size observed with an 270 increased gelatine ratio. This is the likelihood of increased gelatine component of the hydrogel, 271 producing larger droplets during the w/o emulsification and subsequent hardening after the addition 272 of glutaraldehyde. The rotary evaporation method yielded significantly larger particle sizes in 273 comparison to vacuum drying. Interestingly, a positively skewed particle size distribution was 274 observed for all lidocaine hydrogel formulations (Fig. 2).

\section{Fig. 2}

\subsection{Dispersion of lidocaine NaCMC/gel hydrogel microparticles}

Zeta potential studies in lidocaine $\mathrm{NaCMC} /$ gel hydrogels demonstrated a stable and fairly dispersed microparticulate system. The results (Fig. 3) expressed a trend of decreasing stability with an increase in the gelatine ratio, which in theory should impact a greater level of microparticle agglomeration thus likely affecting the permeability through skin. The $\mathrm{pH}$ of all formulations was kept constant and therefore it should not have affected the zeta potential although the slight decline of $\zeta$ potential in the positive direction is linked to the increase in gelatine ratio caused by gelatine in conjunction to lidocaine possessing a positively charged tertiary amide group at $\mathrm{pH} 4.0$ and thus contributing to the increasing negative surface charge. The anionic polymer, sodium carboxymethyl cellulose has a $\zeta$-potential value of $-30 \mathrm{mV}$ (Ducel et al., 2004) and electric charge neutralisation did not occur or was not significantly induced by gelatine or lidocaine, so the overall lidocaine $\mathrm{NaCMC}$ :gel hydrogel charge was greater than $-30 \mathrm{mV}$. Nevertheless, reduced agglomeration is the result of a medium pKa, higher dielectric constants in comparison to a polymeric hydrogel components converging to significantly low overall $\zeta$-potential range of -35 to $-40 \mathrm{mV}$ and effect of electrostatic particle repulsion (Xu et al., 2007).

\section{Fig. 3}

\subsection{Viscoelasticity of lidocaine NaCMC/gel hydrogel}

Viscosity determination (Fig. 4) revealed a lenient pseudoplastic nature for the formulation with lidocaine $\mathrm{NaCMC/gel} \mathrm{hydrogel} \mathrm{with} \mathrm{good} \mathrm{correlative} \mathrm{best} \mathrm{fit} \mathrm{curves} \mathrm{observed} \mathrm{for} \mathrm{individual} \mathrm{set} \mathrm{of}$ data points $\left(R^{2}>0.93\right)$. The dynamic viscosity plots showed similar mild pseudoplastic behaviour between the formulations with lidocaine $\mathrm{NaCMC/gel} \mathrm{1:2.66} \mathrm{hydrogel} \mathrm{being} \mathrm{marginally} \mathrm{higher} \mathrm{when}$ considering the upper viscosity range of 0.5 to $0.6 \mathrm{~Pa}$.s at a starting shear of $25 \mathrm{~s}^{-1}$ and then more defined shear thinning behaviour observed above $100 \mathrm{~s}^{-1}$. Lidocaine with sodium carboxymethylcellulose as a polyanionic vehicle alone will not be sufficient in enhancing 
pseudoplastic properties and a recent study has shown that the profile of a dynamic viscosity plot is Newtonian (Alaie, 2013).

3.4 Control of lidocaine NaCMC:gel 1:2.33 spreading on porcine skin.

309 The spreading radius and height of lidocaine NaCMC/gel 1:2.33 outline significant control on its 310 spreading behaviour compared with lidocaine solution of the same mass loading (Fig. $5 a$ and 5b).

311 The beginning of the plateau effect is observed after 10 seconds and therefore, there is expected to 312 be a localisation effect on the skin surface (Fig. $5 a$ and $5 b$ ). The apparent contact angles of 313 lidocaine $\mathrm{NaCMC} / \mathrm{gel}$ 1:2.33 droplets are considerably higher than the lidocaine solution contact 314 angle droplets, near to the skin impact time of 0 seconds (Fig 5b). Apparent contact angle stability is 315 noticed after 40 seconds (Fig. 5c). Our results also show that the lidocaine solution is a Newtonian liquid that can spread a much faster than lidocaine NaCMC/gel microparticles.

\subsection{The percentage release of lidocaine from controlled release of lidocaine.}

All four lidocaine $\mathrm{NaCMC/gel} \mathrm{hydrogels} \mathrm{outline} \mathrm{rapid} \mathrm{release} \mathrm{of} \mathrm{lidocaine} \mathrm{directly} \mathrm{in} \mathrm{DI} \mathrm{water} \mathrm{during}$ the first 1 hour with steady state conditions observed in the next three hours (Fig. 6a). A 0.3 fold decrease in cumulative release is observed in the first hour when comparing lidocaine $\mathrm{NaCMC/gel}$ 1:1.6 with lidocaine $\mathrm{NaCMC/gel} \mathrm{1:2.66} \mathrm{as} \mathrm{the} \mathrm{highest} \mathrm{releasing} \mathrm{outline.} \mathrm{Also,} \mathrm{a} 0.1$ fold decrease in cumulative release was observed in the next three hours when comparing lidocaine $\mathrm{NaCMC} / \mathrm{gel}$ 1:1.6 with lidocaine $\mathrm{NaCMC/gel} \mathrm{1:2.66.} \mathrm{This} \mathrm{shows} \mathrm{that} \mathrm{the} \mathrm{variation} \mathrm{between} \mathrm{hydrogel} \mathrm{ratios} \mathrm{is} \mathrm{not}$ significantly large as permeation release profiles explained in the following sections. The percentage release of lidocaine from $\mathrm{NaCMC/gel} \mathrm{hydrogels} \mathrm{were} \mathrm{determined} \mathrm{by} \mathrm{the} \mathrm{following} \mathrm{equation} 1$ :

Percentage drug release $=\frac{M s-M t}{M s} \times 100$

329 Where $\mathrm{Ms}$ is the maximum mean cumulative steady state concentration of drug and $\mathrm{Mt}$ is the mean 330 cumulative concentration of lidocaine taken specifically at release time. The highest amount of 331 Lidocaine released was from NaCMC/gel 1:1.6 hydrogel in which $32.3 \%$ was detected in the DI water media in one hour (Fig. 6b). This is because the smaller particles sizes of Lidocaine $\mathrm{NaCMC/gel} \mathrm{1:1.6} \mathrm{ratio} \mathrm{allow} \mathrm{for} \mathrm{a} \mathrm{greater} \mathrm{surface} \mathrm{area} \mathrm{and} \mathrm{encapsulated} \mathrm{lidocaine} \mathrm{thus} \mathrm{rapidly}$ dissolves in $\mathrm{DI}$ water. The lidocaine $\mathrm{NaCMC/gel} \mathrm{1:2.66} \mathrm{ratio} \mathrm{comprises} \mathrm{larger} \mathrm{microparticles} \mathrm{and}$ therefore a smaller surface area is exposed for DI water dissolution so the percentage of lidocaine released was $17.4 \%$ in one hour. Significantly less amounts of lidocaine is released for all $\mathrm{NaCMC/gel} \mathrm{hydrogel} \mathrm{formulations} \mathrm{after} 1$ hour reflecting the steady state conditions of the hydrogel as the DI water media becomes a saturated solution. 


\subsection{Histological analysis on the microneedles}

342 The microneedles that are employed in the histological experiment are $1100 \mu \mathrm{m}$ in length. The

343 purpose of the histological experiment is to determine the insertion depth of this microneedle patch 344 under thumb pressure for which post-microneedle treated skin is micrograph imaged (Fig. 7). 345 According to Fig.7, the insertion depth is between $300 \mu \mathrm{m}$ and $400 \mu \mathrm{m}$ which are much lower than 346 the real length of microneedles. This is caused by several reasons, such as the viscoelastic 347 properties of the skin, the geometry of the microneedles and the insertion force. This reduced 348 insertion depth can further affect the permeation results.

\subsection{Passive diffusion of lidocaine NaCMC/gel hydrogel}

352 Skin passive diffusion experiments were carried out in order to provide a control from which any pre353 treatment enhancement results can be compared and contrasted. The lowest polymeric 354 microparticle ratio 1:1.6 of lidocaine (Fig. 8a) outlines the most desirable cumulative permeation for 355 lidocaine in crossing the minimum threshold therapeutic level after 0.57 hours. This is the shortest 356 lag time for reaching the pain receptors for lidocaine in the deep dermis region rich in watery plasma 357 and nerves. The hydrogel microparticle chemistry is a combination of significantly high negative zeta 358 potential and smaller mean particle size contributing to an increased permeation. All lidocaine $359 \mathrm{NaCMC} / g e l$ ratio hydrogels have demonstrated a very low initial permeation at a maximum of $3600.3 \mu \mathrm{g} / \mathrm{ml}$ reached in 0.5 hours. This is the normal lag time because of a longer path length for 361 microparticle permeation when considering the topmost SC layer surface area bigger than the 362 accessible VE layer microcavities. However, lidocaine NaCMC/gel 1:2.0 and lidocaine NaCMC/gel 363 1:2.66 hydrogels are the next two favourables after the most desirable formulation containing a 364 polymeric mass ratio 1:1.6 for bypassing the minimum therapeutic threshold at a shorter time interval. Initially, lidocaine is diffusing through the fresh skin because of microparticulate disruption to the hydrogel formula caused by natural skin moisture hence the low initial concentration rates proceeding upto 0.5 hours. Due to the requirements of lidocaine as an fast acting anaesthetic the current results confirm enhancement of permeation is required if minimum therapeutic threshold of lidocaine $(1.5 \mu \mathrm{g} / \mathrm{ml})$ are to be reached within a suitable time frame for this technique to be of practical use. The lag time to cross a minimum therapeutic level is slightly greater than 1 hour in

371 lidocaine NaCMC/gel 1:2.33 hydrogel and just over 2 hours for lidocaine NaCMC/gel 1:2.66 372 hydrogel, rotary evaporation method with respect to passive diffusion alone which is considerably a 373 long, unreasonable waiting time for a promising polymeric hydrogel ointment drug. The cumulative 374 lidocaine thresholds tend to stabilise post 4 hours, where equilibrium is reached and no more drug is 
375 released into the concentrated dermal region. This means that the lidocaine hydrogel ointment can 376 be washed off the skin. Lidocaine $\mathrm{NaCMC/gel}$ hydrogel was compared with lidocaine solution permeation from literature (Sekkat et al., 2004). Prior to this passive diffusion comparison with lidocaine solution passive diffusion, the permeation units of $\mu \mathrm{g} / \mathrm{ml}$ were converted into $\mu \mathrm{g} / \mathrm{cm}^{2}$ by the product of the known receptor volume followed by the quotient of the adjustment factor value of 2.36 $\left(3.14 \mathrm{~cm}^{2} / 1.33 \mathrm{~cm}^{2}\right)$ due to the increase in FDC diffusion area when comparing a similar study using a smaller aperture diameter (Sekkat et al., 2004). The current lidocaine NaCMC/gel 1:1.6 hydrogel crosses the minimum therapeutic threshold by 1.8 fold than lidocaine solution on similar full thickness skin despite lidocaine solution permeating initially at 1.4 fold faster before a half an hour time frame and not anywhere near the minimum therapeutic threshold (Sekkat, 2004). Lidocaine NaCMC/gel 1:2.66 and lidocaine NaCMC/gel 1:2.66 hydrogel formulated by rotary evaporation were chosen to be studied for further enhancement via pre-treatment. The factor of permeation enhancement can be deduced when making this comparison.

\subsection{Ultrasound only pre-treatment of lidocaine NaCMC/gel ratio 1:2.66 hydrogel}

To observe the effect of power and application time of LFS has on permeation, LFS was applied continuously with varying power and exposure time as shown in Fig. 8b. Theoretically, the exposure of LFS should form inertial cavities in the coupling medium and develop micro-jets toward the skin surface to aid permeation. However, lidocaine transport through the skin saw no significant enhancement up to 2 hours after which a significant enhancement, especially power induction, $18 \mathrm{~W}$ at 10 mins for lidocaine $\mathrm{NaCMC} /$ gel 1:2.66 (T-test $\mathrm{P}<0.026$ ) outlined a greater permeation profile. The results conclude that an increase in power has a greater enhancement effect compared to an increase in LFS exposure time; however, no significant increase in lidocaine transport through the skin was observed during the initial stages after varying respective power induction and time durations while maintaining constant $\mathrm{NaCMC/gel} \mathrm{ratios} \mathrm{of} \mathrm{lidocaine} \mathrm{hydrogel} \mathrm{drug} \mathrm{application.} \mathrm{It} \mathrm{is}$ predicted that a higher LFS power level would further increase diffusion; however, the risk of thermal effects would be too high for this to be of practical use.

\subsection{Microneedle pre-treatment of lidocaine NaCMC/gel ratio 1:2.66 hydrogel}

PD permeation (Fig. 8d) and microneedle assisted (MN) permeation (Fig. 8c) with a post application time limit of 3 and 5 minutes concurrently were compared altogether. Microneedle only pretreatment of lidocaine $\mathrm{NaCMC/gel} \mathrm{1:2.66}$ hydrogel generated a substantial increase in lidocaine permeation for both the 3 and 5 minute post $\mathrm{MN}$ duration (Fig. 8c). A statistically significant difference $(P<0.04)$ was observed for $M N$ application duration. Initial $(t=0.5 h)$ permeation for the 3 and 5 minute patch duration resulted in increases of 9 and 17 fold respectively. An average 3 fold increase in permeation was observed for the 3 minute microneedle application and comparatively an increase by 4 fold for a 5 minute microneedle application. The results indicate that therapeutic 
413 levels of lidocaine could be reached within 0.15 hours or 9 minutes post application MN, in 414 comparison to no pre-treatment requiring 40 minutes (Fig. 8c, 8d). The reason for this short lag time 415 is due to lidocaine microparticles traveling at a shorter path length to the deep dermis layer. The 416 stratum corneum layer has been bypassed by artificial microneedle cavities. Microneedle assisted 417 cumulative release study with respect to lidocaine formulations has not been performed ex vivo to 418 date. However, in vivo release studies have been performed using non degrading polymeric 419 microneedle array coating of lidocaine alone, sustained approximately 15 minutes of delivery thus 420 proven successful for rapid emergency anaesthesia (Zhang et al., 2012). In vivo release studies 421 with ex vivo cumulative release studies are completely incomparable due to obvious differences in 422 experimental procedures and removal of active drug for characterisation. The lidocaine NaCMC/gel 423 1:1.6 ratio (Fig. 8a) hydrogel crosses the therapeutic level at significantly slower time duration, 424 greater than 30 minutes in lidocaine $\mathrm{NaCMC} /$ gel 1:2.66 ratio in comparison of microneedle and LFS 425 treatment. This is due to the fact that microneedles and ultrasound are involved in either cavity 426 engulfing of larger sized hydrogel microparticles.

3.10 Microneedle and ultrasound (dual) pre-treatment of lidocaine NaCMC/gel ratio 1:2.66 hydrogel

429 Both pre-treatments (dual) were combined and studied for further permeation enhancement in 430 comparison to microneedle or LFS pre-treatment only. Lidocaine NaCMC/gel 1:2.66 hydrogel in 431 which combining a 10 minute application of $18 \mathrm{~W}$ LFS after a 5 minute application of microneedles 432 demonstrated an initial faster permeation by 23 fold with an average 4.8 fold increase over 30 433 minutes of application when compared with separate device treatments and passive diffusion (Fig. 434 8d). Therapeutic levels of lidocaine could theoretically be reached after 7 minutes post application in terms of reaching the deep dermis layer of skin as the target. A general increase in permeation throughout the period of experimentation can be noticed rather than post 2 hours as seen with LFS pre-treatment only, this could be due to efficiency of LFS pre-treatment is further enhanced on porous skin sample formed via the microneedle patch.

\subsection{Dual pre-treatment of lidocaine NaCMC/gel 1:2.66 hydrogel via rotary evaporation method}

441 Lidocaine NaCMC/gel 1:2.66 hydrogel with the rotary evaporation method as described earlier, 442 favoured an additional time of nearly 0.9 hour or fifty minutes after the application of $18 \mathrm{~W}$ LFS at 10 443 minutes $(P<0.04)$ to reach minimum therapeutic level in conjunction to a two fold average increase 444 in permeation after 1 hour, compared with the same formulation without rotary evaporation method 445 (Fig. 8d, 8e). This was the likelihood of higher heating temperatures compromising the 446 glutaraldehyde fixation and thus resulting in larger microparticle as previously reported. Higher 447 heating temperatures were required in the large volume removal of $n$-hexane and paraffin oil 448 mixture by solvent evaporation. A 5 minute application of the microneedle array led to an initial 449 increase by 2.8 fold and subsequently an average 3.4 fold increase was observed with respect to 450 the deep dermis layer skin target. Combining the two pre-treatments resulted in an initial permeation 
451 increase by 3.8 fold followed by an average increase by 4.1 fold in comparison to passive diffusion 452 only (Fig. 8d, 8e). Therapeutic levels of lidocaine were reduced from just over 2 hours to less than 1 453 hour on average.

\subsection{Mass transfer of lidocaine from NaCMC/gel 1:2.66 hydrogel}

455 The percentage of lidocaine remaining inside ex vivo skin was determined by the subtraction of the 456 mass of lidocaine initially encapsulated during formulated preparation $(125000 \mu \mathrm{g})$ by the 457 cumulative amount detected in DI water from controlled release studies. The purpose of using 458 controlled release studies is to determine the amount of lidocaine contained in the vehicle as mass 459 balance before the subtraction of the mass of lidocaine in the receptor in which the DI water in the 460 receptor is the deep dermis. All mass balances were carried out in $\mu \mathrm{g}$ and converted from 461 cumulative concentration units of $\mu \mathrm{g} / \mathrm{ml}$ before the percentage of lidocaine remaining inside the skin 462 was determined (Fig. 9). Overall the mass transfer of lidocaine with respect to all treatment 463 applications appeared to outline a gradual, slow process of diffusing through the full thickness 464 appendage. However there is a fairly substantial decline in the percentage of lidocaine remaining in 465 the skin when microneedle and ultrasound treatment (LFS) method was applied. This can be 466 interpreted as diffusion of lidocaine molecules through skin cells and layers before clearance into 467 the blood stream. The lowest percentage of lidocaine remaining in the skin is $99.7 \%$ after a time of 4683 hours (Fig. 9).

\section{Conclusions}

471 This study aimed to use low frequency sonophoresis and microneedles as a pre-treatment to skin in 472 order to enhance permeation of lidocaine encapsulated in a formulation. A significantly more 473 microparticle stability was found with lower gelatine ratios (1:1.60); however all formulations were 474 sufficiently stable (zeta potential: $\geq-30 \mathrm{mV}$ ). Our diffusion experiments revealed a small increase in 475 diffusional permeation when low frequency sonophoresis was used in combination with a 476 microneedle array pre-treated skin. However, rotary evaporation during the final polymeric drug 477 formulation stage caused significant reductions in lidocaine permeation levels. Nota bene that the 478 main purpose for utilising rotary evaporation was for reduced time in removal of a large volume of 479 residual paraffin and $n$-hexane as the final operative method compared to vacuum oven drying (data 480 not shown). Lidocaine $\mathrm{NaCMC} /$ gel 1:2.66 and lidocaine NaCMC/gel hydrogel 1:2.66 formulated by 481 rotary evaporation showed a decreased time required to reach minimum therapeutic levels of 482 lidocaine by 5.7 and 2 fold, respectively. Generally, lidocaine permeation was significantly increased 483 with higher sonophoresis power and increasing exposure duration demonstrated a minor increase of 484 the permeation rate for lidocaine NaCMC/gel hydrogel formulations. Also the microneedle 485 application time duration of 5 minutes resulted in a highly favourable increase in lidocaine 486 permeation. Furthermore, combining microneedle and low frequency sonophoresis pre-treatments 487 allowed for the time to reach minimum therapeutic lidocaine levels to be significantly reduced, For 
example, in the case of lidocaine $\mathrm{NaCMC} / g e l, 1: 2.66$ hydrogel therapeutic thresholds of lidocaine were reached within 7 minutes of application. The mass transfer effects in which the percentage of lidocaine remained in the full skin depicted the gradual movement of drug in targeting pain receptors below the SC layer. The lidocaine NaCMC/gel 1:2.66 hydrogel treated by microneedles and LFS shows a greater mass transfer profile. The US and MN treated lidocaine NaCMC/gel 1:2.66 has a $0.18 \%$ mass transfer of lidocaine through skin within 2 hours compared with $0.01 \%$ mass transfer of lidocaine through skin. Therefore, this method is promising and could be of medical use as a painless, easy to administer technique for drug delivery overcoming the time constraints associated with delivery of lidocaine. Lidocaine $\mathrm{NaCMC} / g e l$ 1:2.66 hydrogel is likely to be the most desirable drug formulation candidate for further developmental studies reaching potentially important preclinical and final post clinical stage developments. In order to develop a less polydisperse but low micron scale lidocaine hydrogel formulation requires a longer time frame and added investment. The resources and materials in developing a lidocaine NaCMC/gel 1:2.66 hydrogel without rotary evaporation is economical on a batch scale at present. Lidocaine. NaCMC/gel 1:2.33 formulation with defined morphological appearance is able to remain on the surface of the skin for longer durations compared with a lidocaine solution of the same mass loading.

\title{
5. Acknowledgements
}

505

The authors acknowledge the help of Craig Chao for his assistance towards the characterisation of droplet spreading on skin (Figure 5).

\section{References}

509

Alaie, J., Vasheghani-Farahani, E., Rahmatpour, A., Semsarzadeh, MA. (2013). Gelation rheology and water absorption behavior of semi-interpenetrating polymer networks of polyacrylamide and carboxymethyl cellulose. Journal of Macromolecular Science, Part B, 52, 604-613.

Al-Qallaf, B., Das, D.B. (2009). Optimizing microneedle arrays to increase skin permeability for transdermal drug

\author{
delivery.
}

Al Qallaf, B and Das, DB (2008) Optimization of square microneedle arrays for increasing drug permeability in skin, Chemical Engineering Science, 63, 2523-2535, DOI: 10.1016/j.ces.2008.02.007.

Badran M.M, Kuntsche J., Fahr A. (2009) Skin penetration enhancement by microneedle device (Dermaroller ${ }^{\circledR}$ ) in vitro: Dependency on needle size and applied formulation. European Journal of pharmaceutical Sciences, 36, 511-523.

Bal, S.M., Ding, Z., van Riet, E., Jiskoot, W., and Bouwstra, J.A. (2010). Advances in transcutaneous vaccine delivery: Do all ways lead to Rome? Journal of Controlled Release, enables prolonged delivery of naltrexone through microneedle-treated skin. Pharmaceutical Research, 28, 1211-1219. 
526 Benet, L,Z., Oie, S., and Schwartz, J.B. (1996). Design and optimisation of dosage regimens: pharmacokinetic data. In J.G. Hardman, L.E. Limbard, P.B. Molinoff, R.W. Rudon, and A.G. Gilman (Eds.), The pharmacological basis of therapeutics (pp. 1707-1792). McGraw Hill, New York.

Berger, J., Reist, M., Mayer, J.M., Felt, O., Peppas, N.A., and Gurny, R. (2004). Structure and interactions in covalently and ionically crosslinked chitosan hydrogels for biomedical applications. European Journal Pharmaceutics and Biopharmaceutics, 57, 19-34.

Chao TZ, Trybala A., Starov V., Das D.B. (2014). Influence of haematocrit level on the kinetics of blood spreading on thin porous medium during blood spot sampling. Colloids and Surfaces A: Physicochemical and Engineering Aspects, 451, 38-47.

Chen, B., Wei, J., and Iliescu, C. (2010). Sonophoretic enhanced microneedles array (SEMA)— Improving the efficiency of transdermal drug delivery. Sensors and Actuators B: Chemical, 145, 54-60.

Cheung K., Han T., Das D.B. (2014) Effect of force of microneedle insertion on the permeability of insulin in skin. Journal of Diabetes Science and Technology. DOI: 10.1177/1932296813519720 (in press).

Chow KT, Chan LW, Heng PWS (2008) Characterization of spreadability of nonaqueous ethylcellulose gel matrices using dynamic contact angle. Journal of Pharmaceutical Sciences 97: 3467-3481.

Davidson, A., Al-Qallaf, B., and Das, D.B. (2008). Transdermal drug delivery by coated microneedles: Geometry effects on effective skin thickness and drug permeability. Chemical Engineering Research and Design, 86, 1196-1206.

De Boer, A.G., Breimer D.D., Mattie, H., Pronk, J., and Gubbens-Stibbe, J.M. (1979). Rectal bioavailability of lidocaine in man: partial avoidance of "first-pass" metabolism. Clinical pharmacology and therapeutics, 26, 701-709.

Ducel, V., Richard, J., Saulnier, P., Popineau, Y., and Boury, F. (2004). Evidence and characterization of complex coacervates containing plant proteins: application to the microencapsulation of oil droplets. Colloids Surface A. 232, 239-247

Ebrahimi, S., Abbasnia, K., Motealleh, A., Kooroshfard, N., Kamali, F., and Ghaffarinezhad, F. (2012). Effect of lidocaine phonophoresis on sensory blockade: pulsed or continuous mode of therapeutic ultrasound? Physiotherapy, 98, 57-63.

Escobar-Chávez, J.J., Rodríguez-Cruz, I.M., and Domínguez-Delgado, C.L. (2012). Chemical and physical enhancers for transdermal drug delivery. In Dr Luca Gallelli (Ed), Pharmacology,, ISBN: 978-953-51-0222-9

Fasinu, P., Viness Pillay, V., Ndesendo, V.M.K., Du Toit, L.C., and Choonara Y.E. (2011). Diverse approaches for the enhancement of oral drug bioavailability. Biopharmaceutics and drug disposition, 32, 185-209 report and review of the use of lidocaine in airway anesthesia. Pharmacotherapy, 13, 72-78 
Ferrari, M., Desai, T., and Bhatia, S. (2007). BioMEMS and Biomedical Nanotechnology. Vol. 3. New York: Springer.

Gill, H.S., and Prausnitz, M.R. (2007). Coated microneedles for transdermal delivery. Journal of Controlled Release, 117, 227-237.

Giudice E.L., and Campbell, J.D. (2006). Needle-free vaccine delivery. Advanced Drug Delivery Reviews, 58, 68-89.

Ghosh, P., Brogden, N.K., and Stinchcomb, A.L. (2013a). Effect of formulation pH on transport of naltrexone species and pore closure in microneedle-enhanced transdermal drug delivery. Molecular Pharmaceutics, 10, 2331-2339.

Ghosh, P., Pinninti, R.R., Hammell, D.C., Paudel, K.S., and Stinchcomb, A.L. (2013b). Development of a codrug approach for sustained drug delivery across microneedle-treated skin. Journal of Pharmaceutical Sciences, 102, 1458-1467.

González-Rodríguez, M.L., Barros, L.B., Palma, J., González-Rodríguez, P.L., and Rabasco A.M. (2007). Application of statistical experimental design to study the formulation variables influencing the coating process of lidocaine liposomes. International Journal of Pharmaceutics, 337, 336-345.

Grossman J.I., Cooper J.A., Frieden J. (1969) Cardiovascular effects of infusion of lidocaine on patients with heart disease. The American Journal of Cardiology, 24, 191-197.

Guo, L., Qiu, Y., Chen. J., Zhang. S., Xu, B., and Gao, Y. (2013). Effective transcutaneous immunization against hepatitis $B$ virus by a combined approach of hydrogel patch formulation and microneedle arrays. Biomedical Microdevices. DOI 10.1007/s10544-013-9799-z.

Gupta, K.C., and Ravi Kumar, M.N.V. (2000). Semi-interpenetrating polymer network beads of crosslinked chitosan-glycine for controlled release of chlorphenramine maleate. Journal of Applied Polymer Science, 76, 672-683.

Han, T., and Das, D.B. (2013). Permeability enhancement for transdermal delivery of large molecule using low frequency sonophoresis combined with microneedles, Journal of Pharmaceutical Sciences, 102 (10), 3614-3622

Hedge, N.R., Kaveri, S.V., and Bayry, J. (2011). Recent advances in the administration of vaccines for infectious diseases: microneedles as painless delivery devices for mass vaccination. Drug discovery today, 16, 1061-1068.

Herwadkar, A., Sachdeva, V., Taylor, L.F., Silver, H., and Banga, A.K. (2012). Low frequency sonophoresis mediated transdermal and intradermal delivery of ketoprofen. International Journal of Pharmaceutics, 423, 289-296.

Huet, P.M., and Lelorier, J. (1980). Effects of smoking and chronic hepatitis B on lidocaine and indocyanine green kinetics. Clinical pharmacology and therapeutics, 28, 208-215.

Hynynen, K. (2010). MRI-guided focused ultrasound treatments, Ultrasonics, 50, 221-229

Igaki, M., Higashi, T., Hamamoto, S., Kodama, S., Naito, S., and Tokukara. S. (2013). A study of the behavior and mechanism of thermal conduction in the skin under moist and dry heat conditions. Skin Research and Technology, 0, 1-7. 
604 Ito, Y., Ohta, J., Imada, K., Akamatsu, S., Tsuchida, N., Inoue, G., Inoue, N., Takada, K. Dissolving microneedles to obtain rapid local anesthetic effect of lidocaine at skin tissue (2013) Journal of Drug Targeting, 21, 770-775.

Kalluri. H, and Banga, A. (2011). Transdermal delivery of proteins. AAPS PharmSciTech, 12, 431441.

Kim, Y., Park, J., and Prausnitz, M.R. (2012). Microneedles for drug and vaccine delivery. Advanced Drug Delivery Reviews, 64, 1547-1568.

Kochhar, J.S., Lim, W.X.S., Zou, S., Foo, W.Y., Pan, J., Kang, L. Microneedle integrated transdermal patch for fast onset and sustained delivery of lidocaine (2013) Molecular Pharmaceutics, 10, 4272-4280.

Kwon, S.Y. (2004). In vitro evaluation of transdermal drug delivery by a micro-needle patch. Controlled Release Society 31st Annual Meeting Transactions. TheraJect Inc. no. 115.

Lastumäki, T.M., Lassila, L.V.J., and Vallittu, P.K. (2003). The semi-interpenetrating polymer network matrix of fibre-reinforced composite and its effect on the surface adhesive properties. Journal of Materials Science-Materials in Medicine, 14, 803-809.

Li, X., Zhao, R., Qin, Z., Zhang, J., Zhai, S., Qiu, Y., Gao, Y., Xu, B., and Thomas, S,H. (2010). Microneedle pretreatment improves efficacy of cutaneous topical anesthesia. American Journal of Emergency Medicine, 28, 130-134.

Li, X.-G., Zhao, R.-S., Qin, Z.-L., Gao, Y.-H., Zhang, J., Zhai, S.-D., Xu, B. Painless microneedle transdermal patch enhances permeability of topically applied lidocaine (2008) Chinese Journal of New Drugs, 17 (7), pp. 597-601. Merino, G., Kalia, Y.N., DelgadoCharro, M.B., Potts, R.O., and Guy, R.H. (2003). Frequency and thermal effects on the enhancement of transdermal transport by sonophoresis. Journal of Controlled Release, 88, 85-94.

Milewski, M., and Stinchcomb, A. (2011). Vehicle composition influence on the microneedleenhanced transdermal flux of naltrexone hydrochloride. Pharmaceutical Research, 28, 124134.

Mitragotri, S. (2013). Devices for overcoming biological barriers: The use of physical forces to disrupt the barriers. Advanced Drug Delivery Reviews, 65, 100-103.

Mitragotri, S., and Kost, J. (2004). Low-frequency sonophoresis: A review. Advanced Drug Delivery Reviews, 56, 589-601.

Naik. A., Kalia, Y.N., and Guy, R.H. (2000). Transdermal drug delivery: overcoming the skin's barrier function. Pharmaceutical Science and Technology Today, 3, 318-326.

Nayak, A., Das, D.B., and Vladisavljević, G.T. (2013). Microneedle assisted permeation of lidocaine carboxymethylcellulose with gelatine co-polymer hydrogel. Pharmaceutical Research. DOI: 10.1007/s11095-013-1240-z (in press)

Nayak, A., and Das D. (2013). Potential of biodegradable microneedles as a transdermal delivery vehicle for lidocaine. Biotechnolology Letters, 35, 1351-1363 
642 Olatunji, O, Das, DB, Nassehi, V (2012) Modelling transdermal drug delivery using microneedles:

643 Effect of geometry on drug transport behaviour, Journal of Pharmaceutical Sciences, 101(1), pp.164-175, ISSN: 0022-3549. DOI: 10.1002/jps.22736.

Olatunji, O., Das, D.B., Garland, M.J., Belaid, L., and Donnelly, R.F. (2013). Influence of array interspacing on the force required for successful microneedle skin penetration: theoretical and practical approaches. Journal of Pharmaceutical Sciences, 102, 1209-1221.

Olatunji, O, Igwe, CC, Ahmed, AS, Alhassan, OA, Asieba, GO, Das, DB (2014) Microneedles from fish scale biopolymer, Journal of Applied Polymer Science, DOI: 10.1002/app.40377 (in press).

Park, N., Kwon, B., Kim, I.S., and Cho, J. (2005). Biofouling potential of various NF membranes with respect to bacteria and their soluble microbial products (SMP): Characterizations, flux decline, and transport parameters. Journal of Membrane Science, 258, 43-54.

Polat, B., Hart, D., Langer, R., and Blankschtein, D. (2011). Ultrasound-mediated transdermal drug delivery: mechanisms, scope, and emerging trends. Journal of Controlled Release, 152, 330348.

Richards, N., and McMahon, S.B. (2013). Targeting novel peripheral mediators for the treatment of chronic pain. British Journal of Anaesthesia, 111 (1), 46-51.

Roxhed, N., Gasser T.C., Griss, P. (2007) Penetration-enhanced ultrasharp microneedles and prediction on skin intereation for efficient transdermal drug delivery. Journal of microelectromechanical systems, 16, 1429-1440.

Rudin, N.J. (2013). Topical analgesics for chronic pain. Current Physical Medicine and Rehabilitation Reports. DOI 10.1007/s40141-013-0028-8.

Scarfone, R.J., Jasani, M., and Gracely, E.J. (1998). Pain of Local Anesthetics: Rate of Administration and Buffering. Annals of Emergency Medicine, 31 (1), 36-40

Schulz, M., Iwersen-Bergmann, S., Andresen, H., and Schmoldt, A. (2012). Therapeutic and toxic blood concentrations of nearly 1,000 drugs and other xenobiotics. Critical Care, 16, R136

Sekkat, N., Kalia, Y.N., Guy, R.H. (2004). Porcine ear skin as a model for the assessment of transdermal drug delivery to premature neonates. Pharmaceutical Research, 21, 1390-1397.

Shah, U.U., Roberts. M., Orlu Gul, M., Tuleu, C., and Beresford, M.W. (2011). Needle-free and microneedle drug delivery in children: A case for disease-modifying antirheumatic drugs (DMARDs). International Journal of Pharmaceutics, 416,1-11.

Shipton, E.A. (2012). Advances in delivery systems and routes for local anaesthetics. Trends in Anaesthesia and Critical Care, 2, 228-233.

Wilson,

J.R.,

Kehl,

L.J.,

Beiraghi,

S. (2008). Enhanced topical anesthesia of $4 \%$ lidocaine with microneedle pretreatment and iontophoresis. Northwest dentistry, 87, 40-41. 
681 Wolloch L, Kost J (2010) The importance of microjet vs shock wave formation in sonophoresis. Journal of Controlled Release, 148, 204-211.

$683 \mathrm{Xu}, \mathrm{R} ., \mathrm{Wu}, \mathrm{C}$., and Xu, H. (2007). Particle size and zeta potential of carbon black in liquid media.

$684 \quad$ Carbon. $45: 2806-2809$

685 Zhang, Y., Brown, K., Siebenaler, K., Determan, A., Dohmeier, D., and Hansen, K. (2012).

686 Development of lidocaine-coated microneedle product for rapid, safe, and prolonged local 687 analgesic action. Pharmaceutical Research, 29, 170-177.

688 Zhang, Y., Siebenaler, K., Brown, K., Dohmeier, D., and Hansen, K. (2012). Adjuvants to prolong 689 the local anesthetic effects of coated microneedle products. International Journal of $690 \quad$ Pharmaceutics, 439, 187-192.

691 Zhang, D, Das, DB, Rielly, CD (2014) Potential of microneedle-assisted micro-particle delivery by 692 gene guns: a review, Drug Delivery, DOI: 10.3109/10717544.2013.864345.

693 Zhao, Y., Kang, J., and Tan, T. (2006). Salt-, pH- and temperature-responsive semi-interpenetrating 694 polymer network hydrogel based on poly(aspartic acid) and poly(acrylic acid). Polymer, 47, $695 \quad 7702-7710$. 
Table 1

Lidocaine $\mathrm{NaCMC} / g e l$ hydrogel mass ratio with particle size values

\begin{tabular}{cccccccc}
\hline $\begin{array}{c}\text { Sample } \\
\text { ID }\end{array}$ & $\begin{array}{c}\text { NaCMC } \\
(\% \text { w/v })\end{array}$ & $\begin{array}{c}\text { Gelatine } \\
(\mathrm{c} \% \text { w/w })\end{array}$ & $\begin{array}{c}\text { Lidocaine } \\
(\% \text { w/w })\end{array}$ & $\begin{array}{c}\text { NaCMC:Gelatine } \\
\text { ratio }\end{array}$ & $\begin{array}{c}\text { Mean Particle } \\
\text { Drier Type }\end{array}$ & $\begin{array}{c}\text { Particle } \\
\text { Diameter } \pm \text { S.D. } \\
(\mu \mathrm{m})\end{array}$ & $\begin{array}{c}\text { Diameter range } \\
(\mu \mathrm{m})\end{array}$ \\
\hline F1 & 1.2 & 2.0 & 2.4 & $1: 1.6$ & Vacuum & $5.89 \pm 0.0026$ & $1-13$ \\
F2 & 1.2 & 2.4 & 2.4 & $1: 2.00$ & Vacuum & $6.04 \pm 0.0027$ & $1-14$ \\
F3 & 1.2 & 2.8 & 2.4 & $1: 2.33$ & Vacuum & $6.81 \pm 0.0029$ & $2-17$ \\
F4 & 1.2 & 3.2 & 2.4 & $1: 2.67$ & Vacuum & $7.42 \pm 0.0029$ & $3-17$ \\
F5 & 1.2 & 3.2 & 2.4 & $1: 2.67$ & Rotary & $14.60 \pm 0.0067$ & $4-31$ \\
\hline
\end{tabular}


698
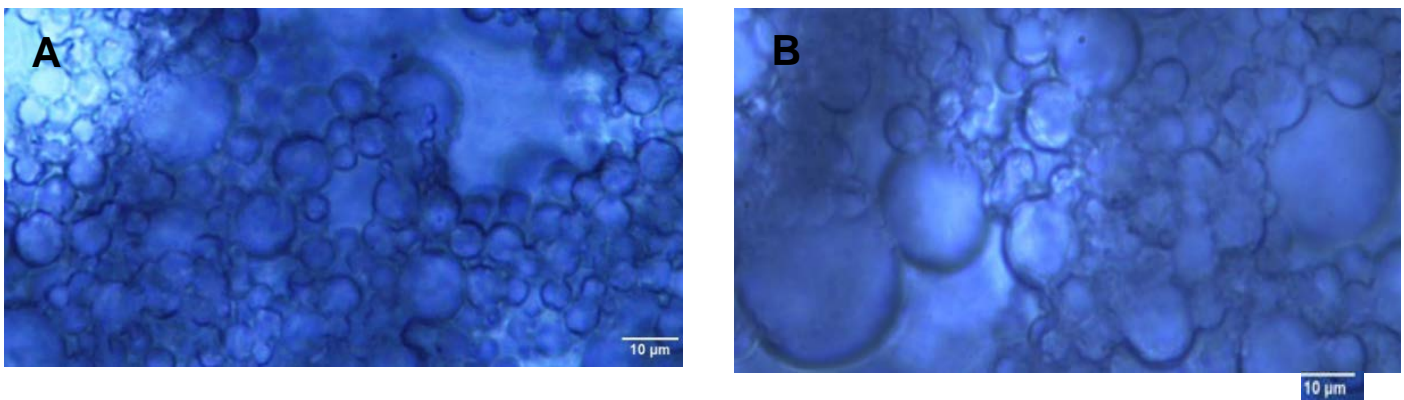

699

Fig. 1. Micrograph of a. lidocaine NaCMC;gel 1:2.33 hydrogel showing distinctly formed microparticles.

700

b. lidocaine NaCMC:gel 1:2.66 hydrogel showing larger and slightly more agglomerated microparticles.

701 


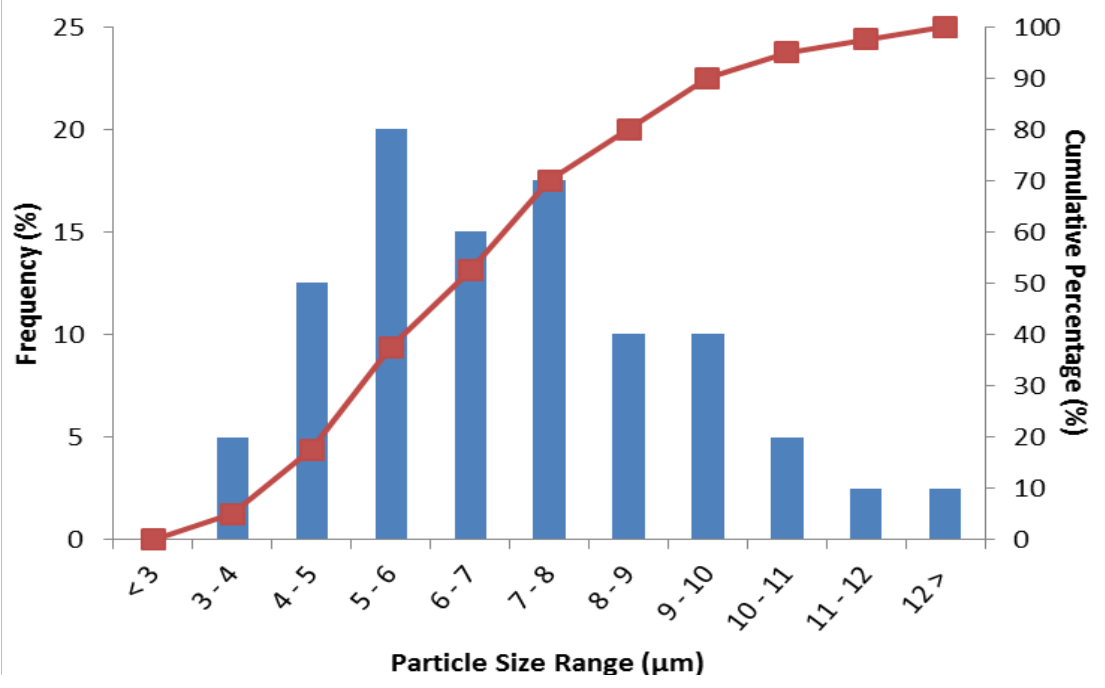

Fig. 2. Particle size distribution of Lidocaine NaCMC/gel hydrogels 


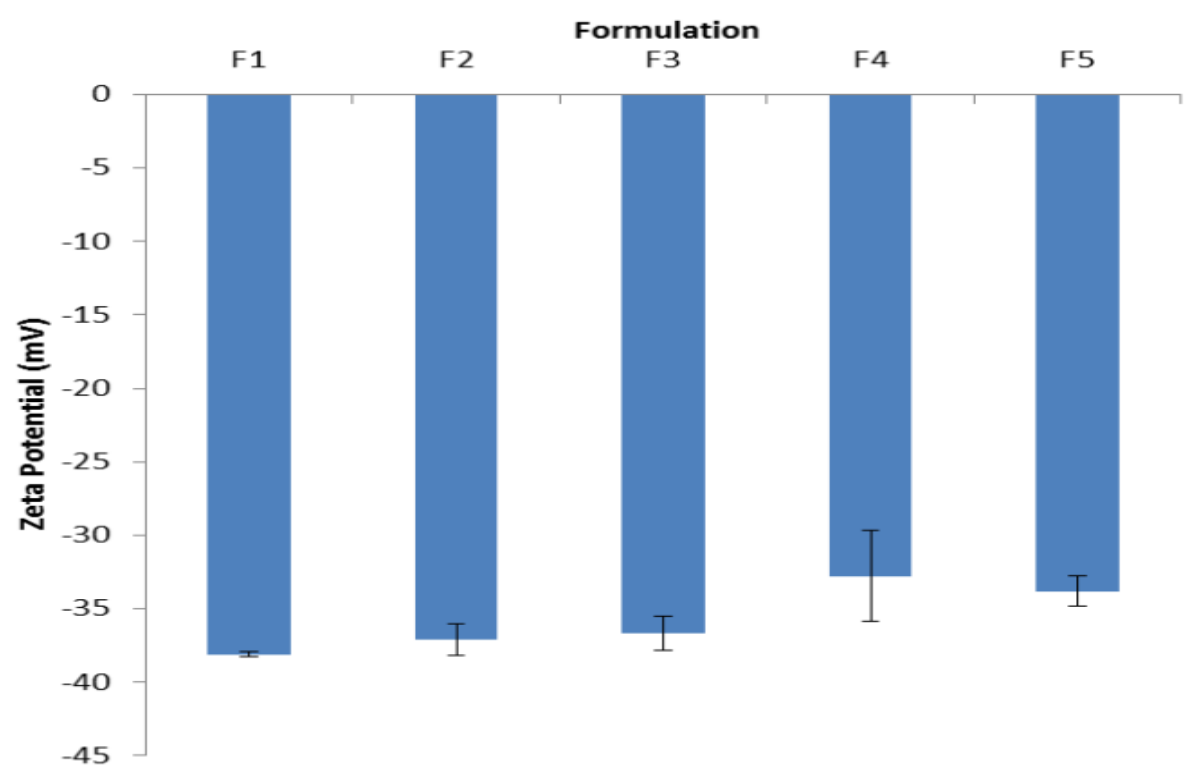

Fig. 3. Lidocaine NaCMC/GEL 1:1.6 to 1:2.66 (F1 to F4) and lidocaine $\mathrm{NaCMC/GEL1:2.66}$ by rotary evap prep. (F5) for zeta potential 


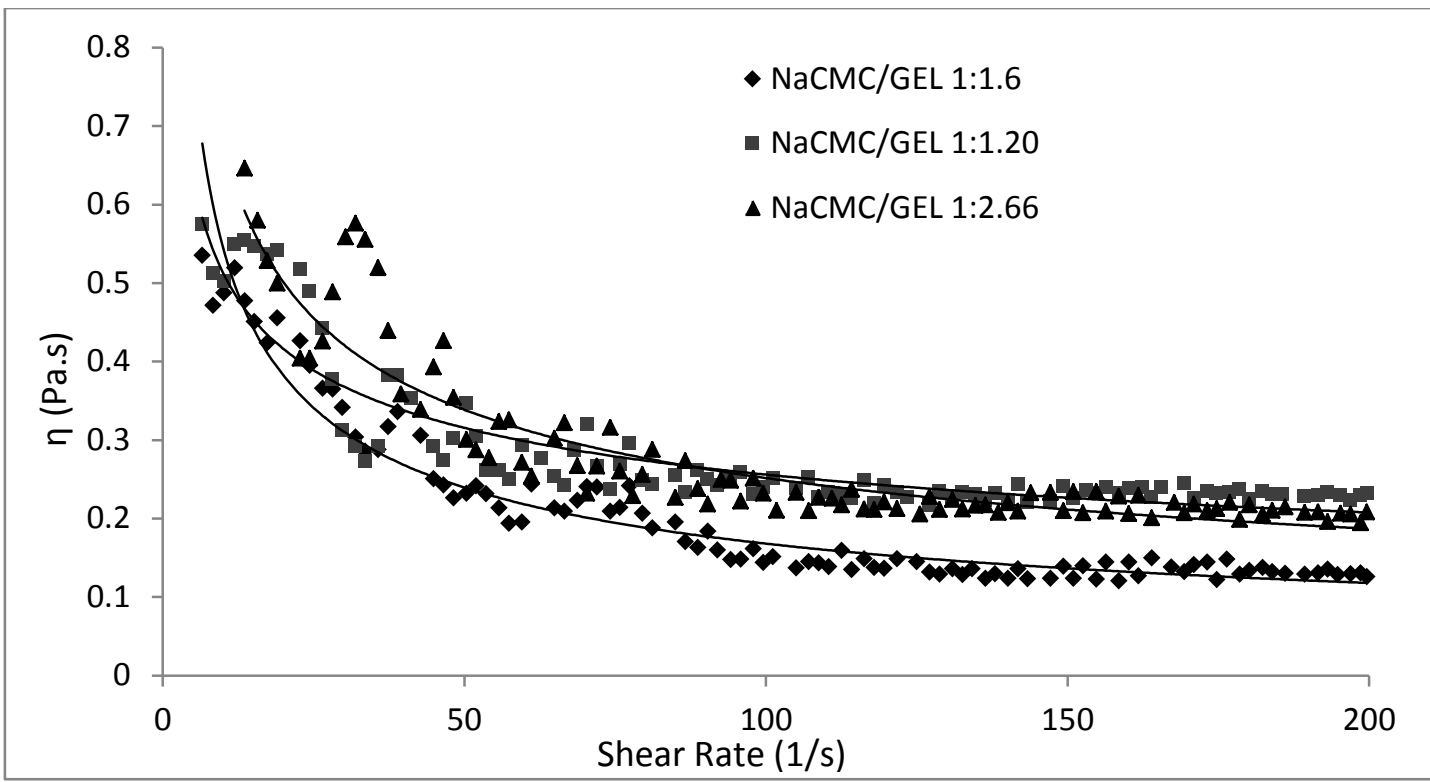

713

Fig. 4. Lidocaine $2.44 \% \mathrm{w} / \mathrm{w}$ NaCMC/GEL ratio pseudoplasticity

714

715

716

717

718

719

720

721

722

723

724

725

726 

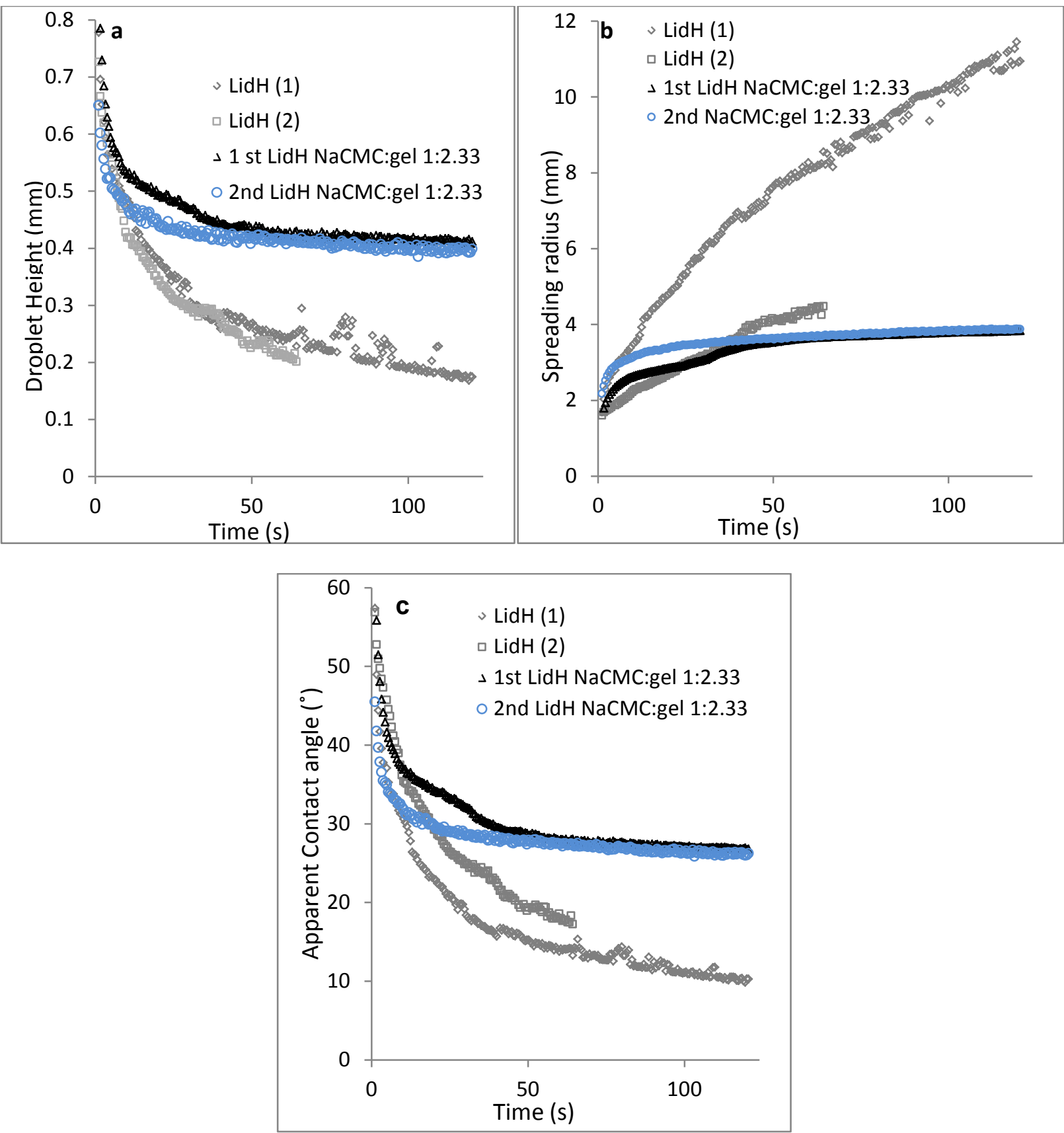

Fig. 5 Lidocaine NaCMC/gel 1:2.33 comparison with Newtonian lidocaine solution according to $\mathbf{a}$. droplet heights $\mathbf{b}$. spreading radii $\mathbf{c}$. apparent contact angles. The results suggest that the spreading of lidocaine $\mathrm{NaCMC/gel} \mathrm{1:2.33} \mathrm{on}$ the skin surface is much more predictable/controllable as compared to lidocaine solution. 

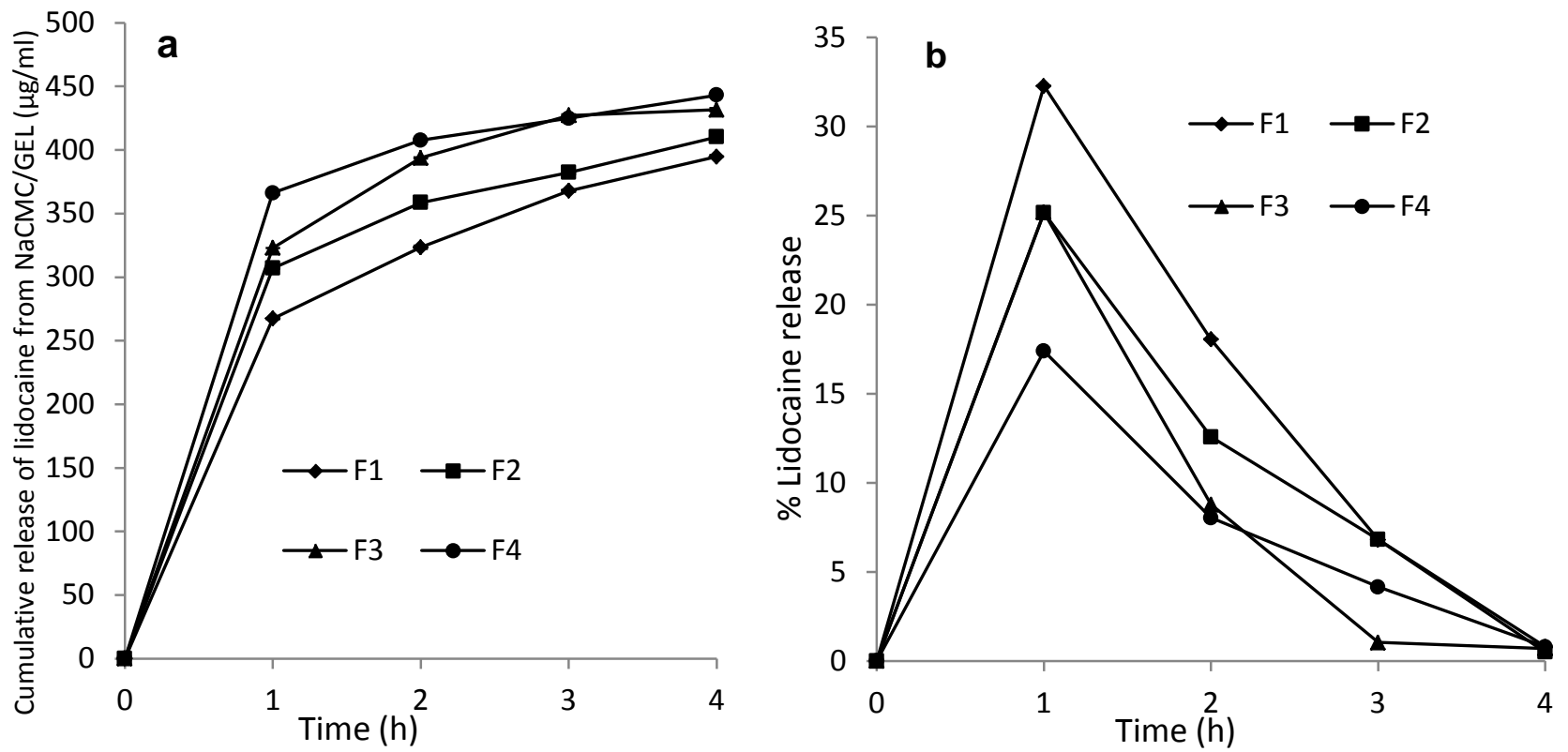

Fig. 6. The controlled release of Lidocaine $2.44 \%$ w/w encapsulated a. NaCMC/GEL 1:1.6 (F1), NaCMC/GEL 1:2.0 (F2), NaCMC/GEL 1:2.33 (F3) and NaCMC/GEL 1:2.66 (F4) b. as a percentage into DI water medium from NaCMC/GEL 1:1.6 (F1), NaCMC/GEL 1:2.0 (F2), NaCMC/GEL 1:2.33 (F3) and NaCMC/GEL 1:2.66 (F4). The error bars in a) the standard deviation of mean represents the error. b) No error bars indicated 
741

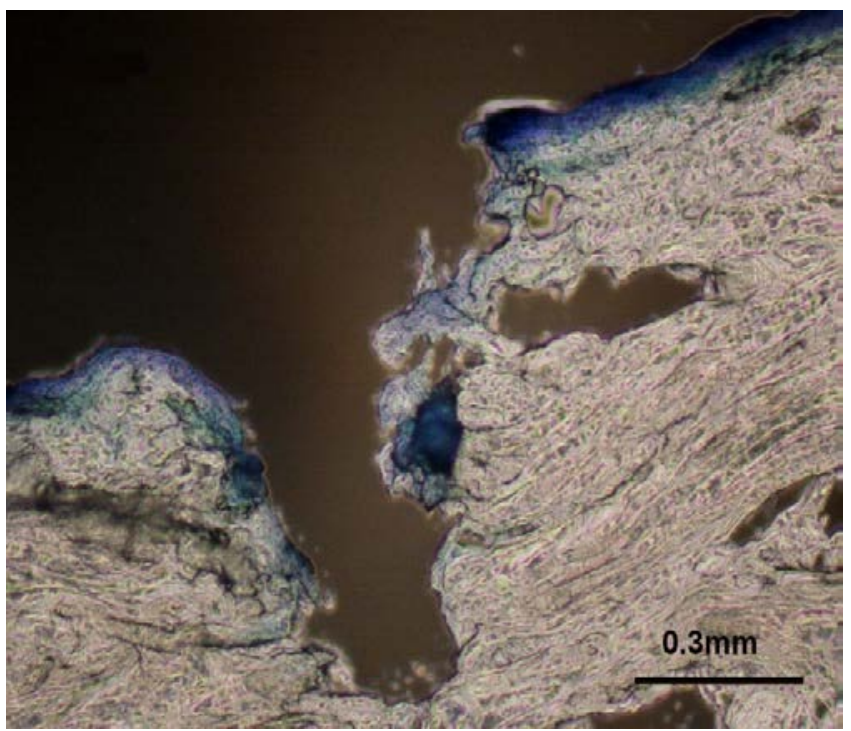

743 Fig. 7 The microneedle insertion depth of skin sample using $1100 \mu \mathrm{m}$ microneedles under thumb 744 pressure. The histological studies shows that although the microneedles are $1100 \mu \mathrm{m}$, for the 745 microneedle density in the array and force applied, they creates holes of approximately $400 \mu \mathrm{m}$. 

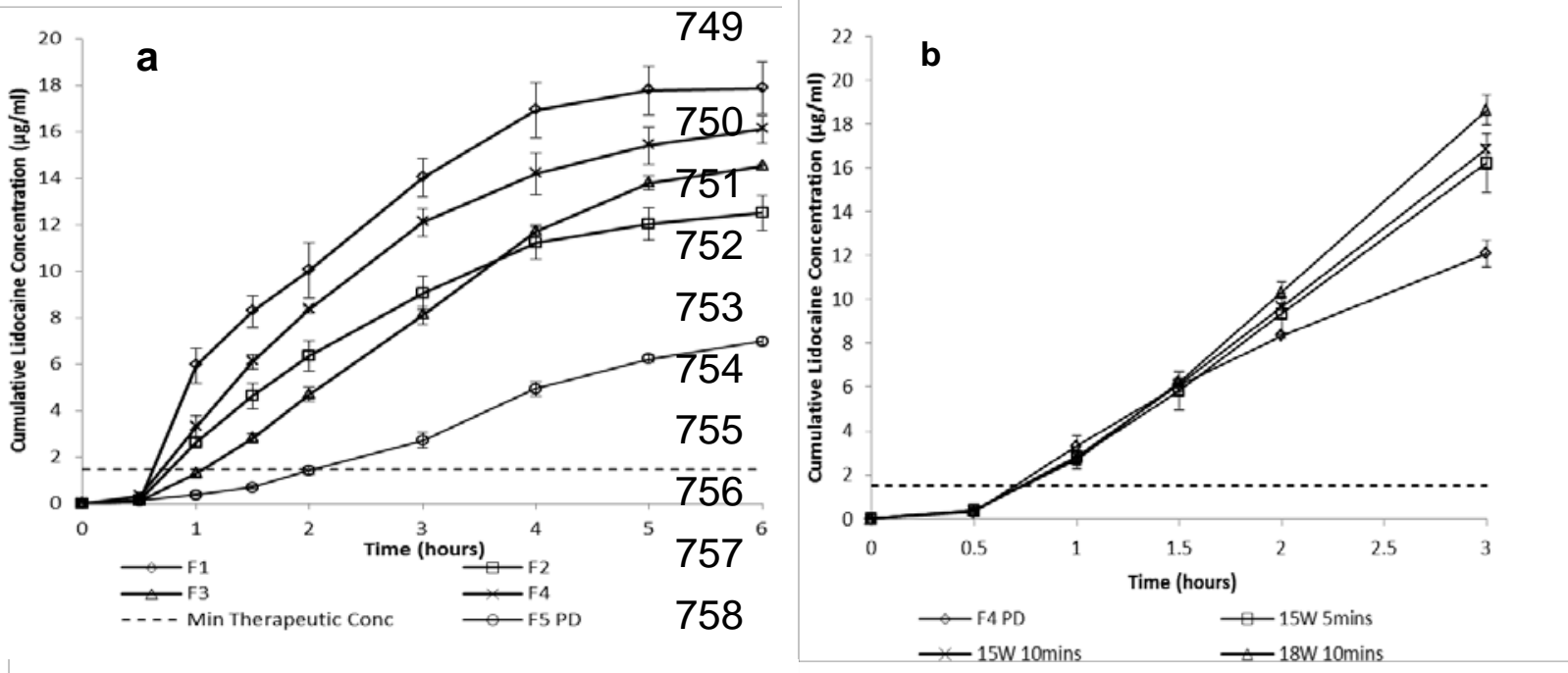

760
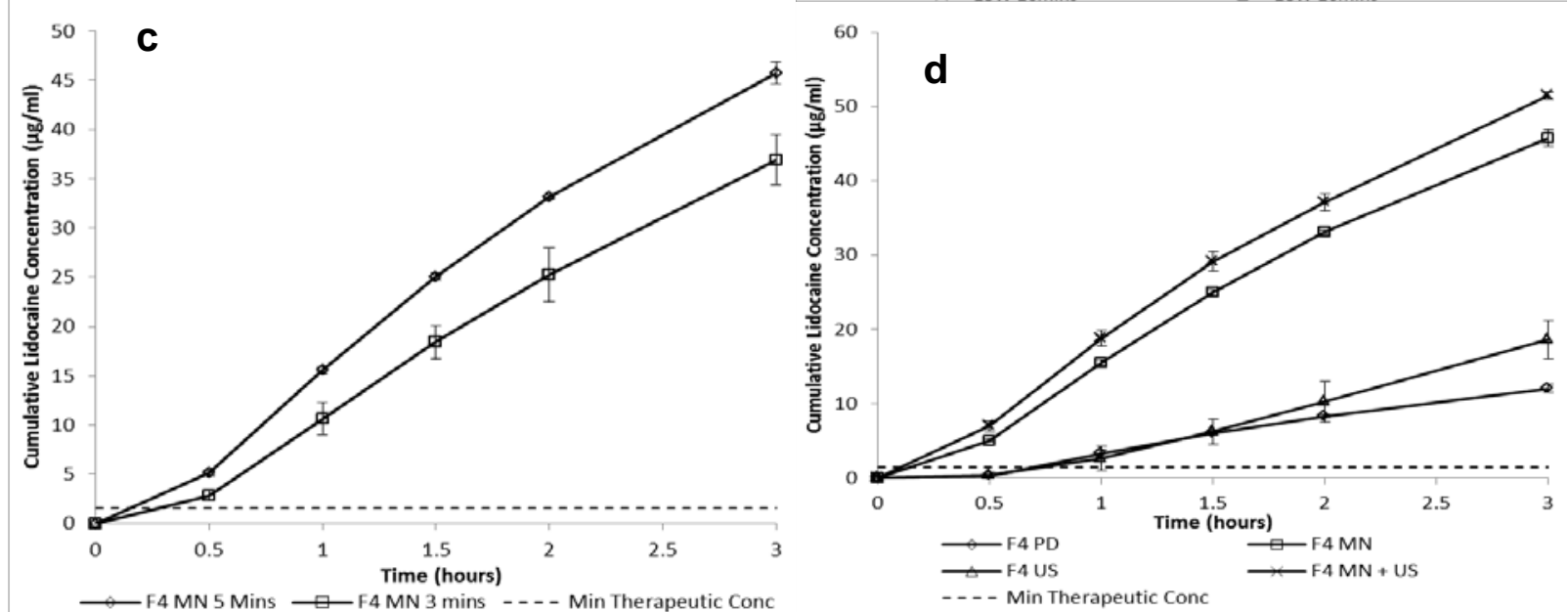

766

767

768

769

770

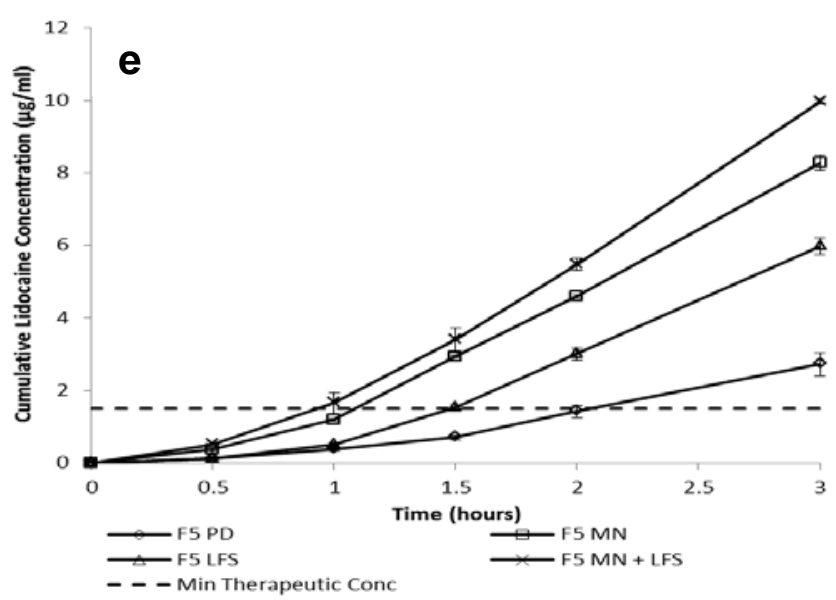

772

Fig. 8. Cumulative lidocaine permeation from Lidocaine. a NaCMC/GEL 1:1.6 (F1), NaCMC/GEL 1:2.0 (F2), NaCMC/GEL 1:2.33 (F3), NaCMC/GEL 1:2.66 (F4) and passive diffusion (PD) NaCMC/GEL 1:2.66 by rotor evaporation prep stage (F5). b F4 PD and comparative pre-treatment with ultrasound at $15 \mathrm{~W}$ and $18 \mathrm{~W}$ for 5 and 10 minutes respectively. c F4 adapting a microneedle (MN) patch for a 3 minute and 5 minute pre-treatment duration for Lidocaine NaCMC/GEL 1:2.66. d F4 adapting NaCMC/GEL 1:2.66 (F4 PD), NaCMC/GEL 1:2.66 (F4 US, 18W 10min.), NaCMC/GEL 1:2.66 (F4 MN, 5 min.) and NaCMC/GEL 1:2.66 (F4 MN 5 min and US 18W 10min). e NaCMC/GEL 1:2.66 (F5 PD), NaCMC/GEL 1:2.66 (F5 LFS, 18W 10min.), NaCMC/GEL 1:2.66 (F5 MN, 5 min.) and NaCMC/GEL 1:2.66 (F5 MN 5 min, LFS, 18W 10min). 
776

777

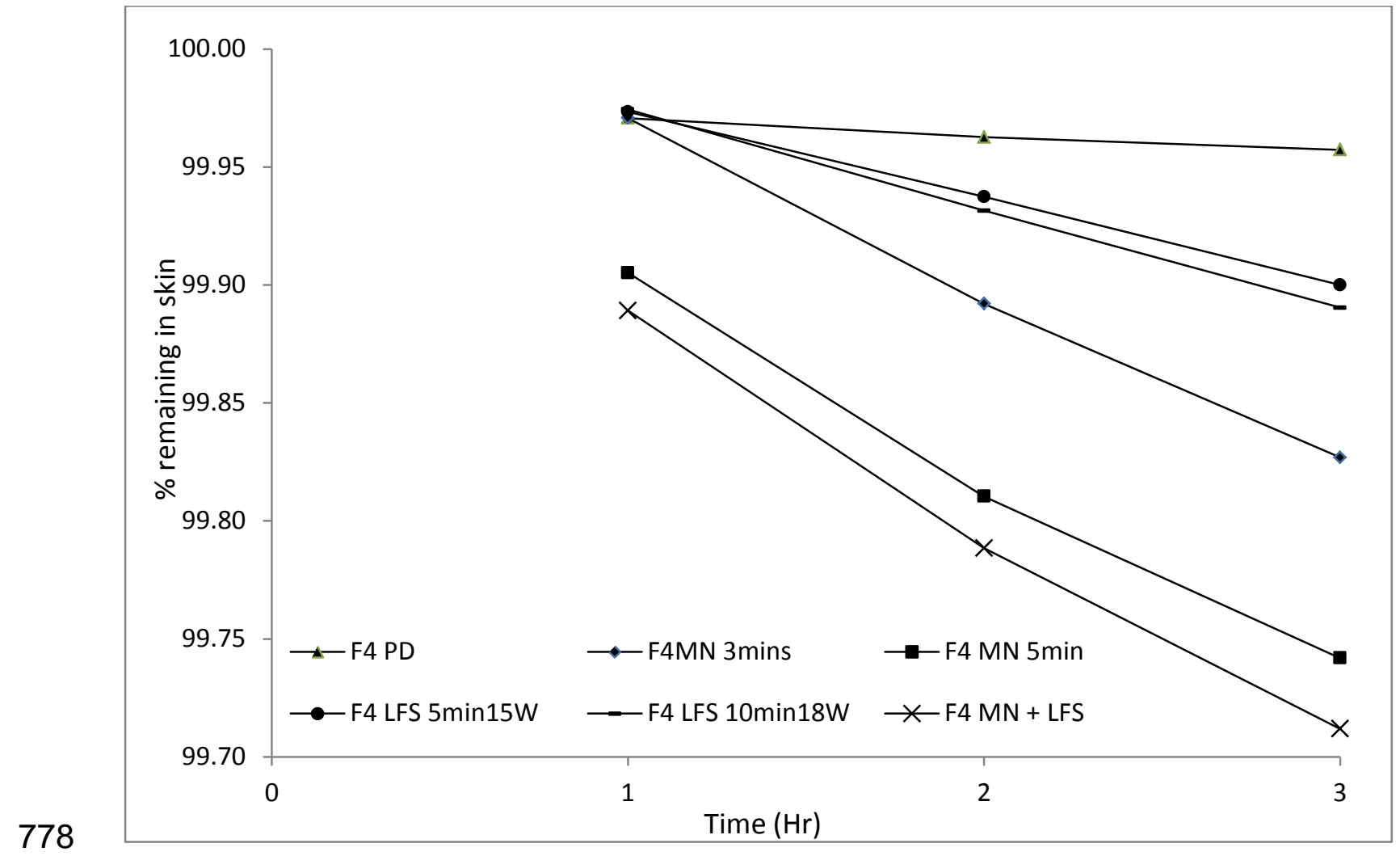

779 Fig. 9. Percentage of lidocaine contained in (F4) NaCMC/GEL 1:2.66 ( $\bullet$ Passive Diffusion), $(\bullet$ microneedles, $3 \mathrm{~min}$ ), 780 ( microneedles, 5 min.), (• LFS 5 min 15W), ( LFS 10 min 18W), ( $\mathbf{* N}+$ LFS). (Error bars outline a 781 random error range of $0.005 \%)$ 